\title{
Bilateral Paget's Disease of the Breast in a Patient with CHEK2 Mutation
}

Nicci Owusu-Brackett (1), Preethi Dileep Menon (1), Alia Nazarullah (), Ismail Jatoi (1), Maryam Elmi (1)

UT Texas Health San Antonio MD Anderson Cancer Center, San Antonio, TX, USA

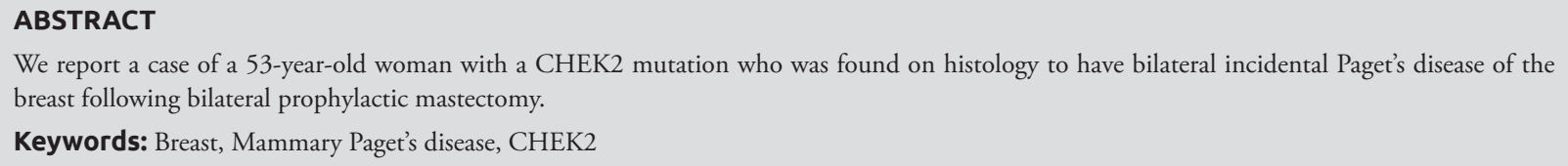

Cite this articles as: Owusu-Brackett N, Menon PD, Nazarullah A, Jatoi I, Elmi M. Bilateral Paget's Disease of the Breast in a Patient with CHEK2 Mutation. Eur J Breast Health 2020; 16(2): 152-154.

\section{Introduction}

CHEK2 is a tumor suppressor gene that encodes a serine/threonine kinase involved in DNA repair, cell cycle arrest or apoptosis as a result of DNA damage. CHEK2 germline mutations have been implicated in numerous types of cancers. In women with the CHEK2 mutation and no family history of breast cancer, the risk of developing breast cancer has been estimated to be as high as $20 \%$, but that risk can be as high as $44 \%$ in women with a family history of breast cancer (1). However, bilateral prophylactic mastectomy is generally not recommended for asymptomatic women with the CHEK 2 mutation, as it is considered a low to moderate penetrance mutation. We now report a case of a woman with CHEK2 mutation and a strong family history of breast cancer who specifically requested bilateral prophylactic mastectomy and was found to have an incidental finding of bilateral mammary Paget's disease on histology. Mammary Paget's disease (MPD) accounts for $1-3 \%$ of new cases of breast cancer diagnosed each year in the United States (1). Paget's disease is commonly unilateral; therefore, bilateral MPD is of even more interest.

\section{Case Presentation}

\section{Clinical history}

A 53-year-old postmenopausal female with a past medical history of duodenal lymphoma presented with bilateral clear nipple discharge and intermittent right nipple bloody discharge of 9 months.

\section{Radiologic investigation}

The patient had a normal screening mammogram the year prior. Upon presenting with new onset of nipple discharge, she underwent a diagnostic mammogram, reported as BIRADS 2 devoid of suspicious masses, adenopathy or of architectural distortion. A few typically benign calcifications were noted bilaterally; however, the breast tissue was noted to be heterogeneously dense, which could obscure detection of small masses. As a result, a bilateral gadolinium contrast-enhanced magnetic resonance imaging (MRI) exam was performed, which showed a $4 \mathrm{~mm}$ oval circumscribed mass in the right breast at 6:00 axis anterior depth, $1.9 \mathrm{~cm}$ from the nipple.

\section{Histopathologic examination}

She underwent an MRI-guided core needle biopsy of the mass using a 9-gauge vacuum-assisted Suros coaxial biopsy device. 9 specimens 
were obtained and submitted to pathology in formalin. The pathology revealed an intraductal papilloma without any evidence of atypia or malignancy.

\section{Genetic examination}

The patient indicated that both her mother and sister, had died of breast cancer, and her sister was found to harbor the CHEK2 mutation. The patient underwent genetic testing and was found to have the same CHEK2 c.1100delC deleterious mutation as reported for her sister.

\section{Management}

The patient requested bilateral prophylactic mastectomy, and a bilateral skin-sparing mastectomy with immediate autologous reconstruction was performed, and the specimen submitted for routine histopathological examination.

\section{Histopathologic examination}

Bilateral mastectomy specimens were submitted for histopathologic examination. There were no grossly identifiable lesions in bilateral breasts and both nipple/areola complex were grossly unremarkable with no scaling, crusting or erythema. Formalin fixed paraffin embedded sections were stained with hematoxylin \& eosin stain for histologic examination. Scattered large atypical cells with round to oval nuclei, vesicular chromatin, prominent nucleoli and abundant pale eosinophilic cytoplasm were noted in the nipple epidermis, predominantly in the basal layers (Figure 1). Some of these atypical cells formed glandular structures with lumen containing focal mucin droplet (Figure 2). The atypical cells were strongly positive for CK7, estrogen receptor, progesterone receptor and negative for HER2/neu by immunohistochemistry (Figure 3). Extensive sampling of the reminder of the breast tissue showed no evidence of in-situ or invasive carcinoma. A diagnosis of bilateral Paget disease of the nipple was rendered.

\section{Discussion and Conclusion}

To the best of our knowledge, this is the first report of bilateral Paget's disease in a CHEK2 mutation carrier.

Clinical Paget's disease which presents as an eczematous or ulcerated lesion on the nipple that spreads to the areola is rare; however, Paget's disease diagnosed on histology is more frequent (2). This lesion may also be associated with pain, pruritus, bloody discharge or nipple retraction. A case series of 3000 mastectomy specimens demonstrate a $0.7 \%$ incidence of clinical Paget disease while histologic evidence of Paget disease was observed in $4.9 \%$ of mastectomies (3). Given that $85-88 \%$ percent of cases of MPD have an underlying breast cancer-

\section{Key Points}

- $\quad$ CHEK2 germline mutations have been implicated in numerous cancers.

- Carriers of CHEK2 deleterious mutations are at increased risk of breast cancer.

- We report the first case of bilateral mammary Paget's disease in a patient with CHEK2 c.1100delC deleterious mutation.

- Further larger series studies are needed to determine the safety of nipple-preservation in CHEK2 mutation carriers.

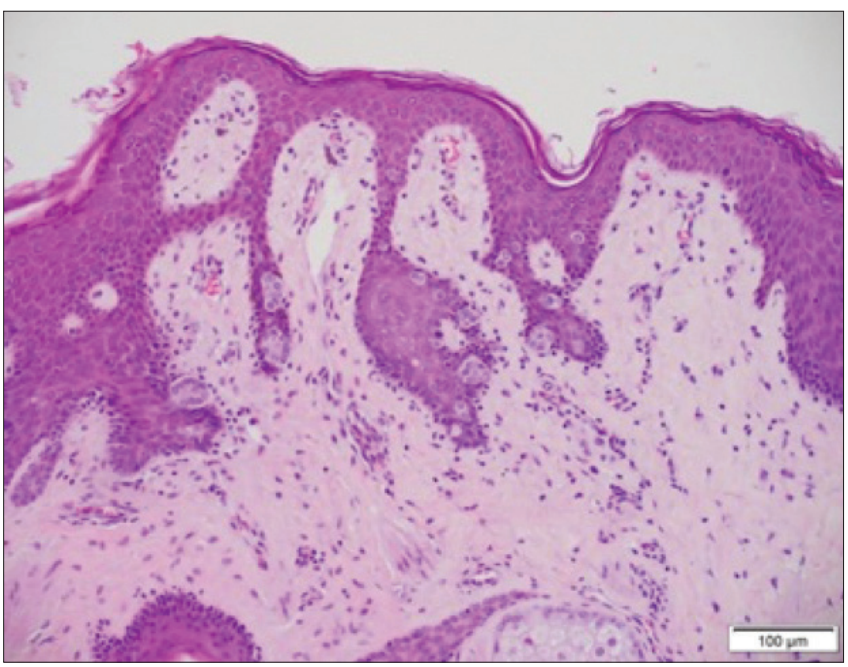

Figure 1. Histologic examination of breast biopsies, where scattered atypical cells were noted in the basal layers of the nipple epidermis

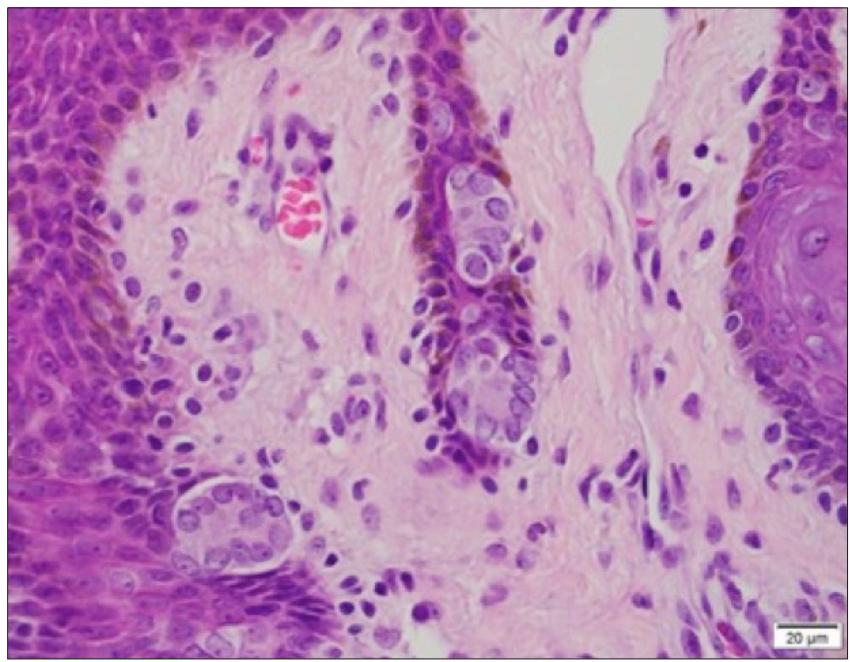

Figure 2. Histologic examination of the same tissue, where the atypical cells were observed to form glandular structures with lumen containing focal mucin droplet

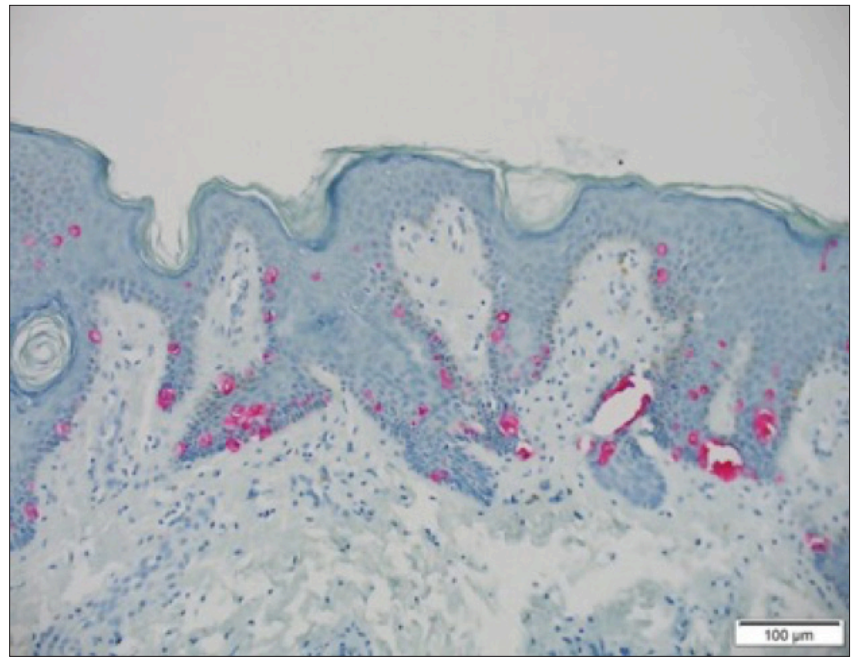

Figure 3.CK7 evaluation of the atypical cells by immunohistochemistry 
invasive or in situ process-, the diagnostic workup of MPD with mammogram and skin or core biopsy focuses on both establishing a diagnosis based on the presence of malignant intraepithelial adenocarcinoma within the epidermis of the nipple and identifying an underlying breast cancer (4). According to the epidermotropic theory, neoplastic ductal epithelial cells migrate from the duct into the epidermis of the nipple; therefore, many cases of MPD have an underlying breast cancer lesion (5).

Whole exome sequencing of MPD has identified frequent recurrent mutations within chromatin remodeling genes such as ARID2 and KMT2C and recurrent somatic mutations such as CDCC168, CASP8AP2, FSIP2 (6). Transcriptome analyses have revealed dysregulation of glandular developmental regulator gene FOXA1; however, CHEK2 mutation has never been described in association with MPD (7). Our patient had CHEK2 c.1100delC mutation. The $1100 \mathrm{delC}$ proteintruncating variant has been associated with a threefold increased risk of breast cancer (8). Research has shown that this breast cancer risk is correlated with family history and the risk increases with first and second-degree relatives with breast cancer.

Standard therapy for MPD is simple mastectomy or breast-conserving therapy. Breast-conservation for MPD entails a central lumpectomy with en bloc excision of the nipple-areolar complex, axillary staging if an invasive component is identified, followed by adjuvant whole breast radiotherapy. The safety of nipple-sparing mastectomy (NSM) is CHEK2 has not yet been studied, and thus, our case report serves as a caution to breast surgeons that NSM may not been indicated in this specific patient population. Further studies (e.g. prospective larger series) are needed to determine the safety of NSM in CHEK2 patients.

Informed Consent: Written informed consent was obtained from patient who participated in this case.

Peer-review: Externally peer-reviewed.

Author Contributions: Concept - N.B., M.D., A.N., I.J., M.E.; Design N.B., M.D., A.N., I.J., M.E.; Supervision - M.E., I.J., A.N.; Resources - N.B.,
M.D., A.N., I.J., M.E.; Materials - A.N., N.B.; Data Collection and/or Processing - N.B., M.D., A.N., I.J., M.E.; Analysis and/or Interpretation - N.B., M.D., A.N., I.J., M.E.; Literature Search - N.B., M.E.; Writing Manuscript - N.B., M.D., A.N., I.J., M.E.; Critical Review - N.B., M.D., A.N., I.J., M.E.; Other - N.B., M.D., A.N., I.J., M.E.

Conflict of Interest: The authors have no conflicts of interest to declare.

Financial Disclosure: The authors declared that this study has received no financial support.

\section{References}

1. Cybulski C, Wokolorczyk D, Jakubowska A, Huzarski T, Byrski T, Gronwald J, et al. Risk of breast cancer in women with a CHEK2 mutation with and without a family history of breast cancer. J Clin Oncol 2011;29: 3747-3752. (PMID: 21876083) [CrossRef]

2. Berg JW, Hutter RV. Breast cancer. Cancer 1995; 75(1 Suppl): 257-269. (PMID: 8001000) [CrossRef]

3. Lagios MD, Gates EA, Westdahl PR, Richards V, Alpert BS. A guide to the frequency of nipple involvement in breast cancer. A study of 149 consecutive mastectomies using a serial subgross and correlated radiographic technique. Am J Surg 1979; 138: 135-142. [CrossRef]

4. Chen CY, Sun LM, Anderson BO. Paget disease of the breast: changing patterns of incidence, clinical presentation, and treatment in the U.S. Cancer 2006; 107: 1448-1158. [CrossRef]

5. Babu B, Dev B, Mohanapriya T, Shalini CNS. Bilateral mammary Paget disease in a young adult female. Radiol Case Rep 2018; 13: 586-591. (PMID: 30008979) [CrossRef]

6. Zhang G, Zhou S, Zhong W, Hong L, Wang Y, Lu S, et al. Whole-Exome Sequencing Reveals Frequent Mutations in Chromatin Remodeling Genes in Mammary and Extramammary Paget's Diseases. J Invest Dermatol 2019; 139: 789-795. (PMID: 30905357) [CrossRef]

7. Mai R, Zhou S, Zhou S, Zhong W, Hong L, Wang Y, et al. Transcriptome analyses reveal FOXA1 dysregulation in mammary and extramammary Paget's disease. Hum Pathol 2018; 77: 152-158. (PMID: 29630912) [CrossRef]

8. Weischer M, Nordestgaard BG, Pharoah P, Bolla MK, Nevanlinna H, Van't Veer LJ, et al. CHEK2*1100delC heterozygosity in women with breast cancer associated with early death, breast cancer-specific death, and increased risk of a second breast cancer. J Clin Oncol 2012; 30: 43084316. (PMID: 23109706) 